\title{
Tunneling properties of quantum dot arrays in a strong magnetic field
}

\author{
V. Moldoveanu, ${ }^{1}$ A. Aldea, ${ }^{1}$ and B. Tanatar ${ }^{2}$ \\ ${ }^{1}$ National Institute of Materials Physics, P.O. Box MG-7, Bucharest-Magurele, Romania \\ ${ }^{2}$ Department of Physics, Bilkent University, Bilkent, 06800 Ankara, Turkey
}

(Received 2 February 2004; revised manuscript received 19 April 2004; published 9 August 2004)

\begin{abstract}
We study the transport properties of coherently coupled quantum dots in the quantum Hall regime within the Landauer-Büttiker formalism which captures and explains the experimentally observed features in terms of the spectral properties of the coupled dot system. The subpeak structure of the transmittance spectrum and the charging stability diagrams are obtained and discussed. The role of the intradot and interdot Coulomb interaction are pointed out. We show the subpeak evolution with the magnetic field and predict a specific oscillatory behavior of the Hall resistance in strong magnetic field which can be experimentally tested.
\end{abstract}

DOI: 10.1103/PhysRevB.70.085303

PACS number(s): 73.23.Hk

\section{INTRODUCTION}

The electronic transport through coupled quantum dots (CQD) became a topic of interest once the basic phenomena in single dots were satisfactorily understood and the experiments on CQD revealed a number of physical effects not encountered in the case of single dots (see Ref. 1 for a review). Waugh et al. ${ }^{2,3}$ investigated experimentally at zero magnetic field the evolution of Coulomb oscillations of the conductance of double and triple quantum dots as the coupling between them (i.e., the interdot conductance) is varied. They found that a usual conductance peak splits into two or three subpeaks (a multiplet) as the coupling strength increases. When the interdot coupling approaches $2 e^{2} / h$ the multiplets merge to distinct peaks, resembling the Coulomb oscillations of a single large dot. This process is the so-called saturation effect discussed by Waugh et al. ${ }^{2}$ The next step was accomplished by Livermore et al. ${ }^{4}$ who measured the conductance through coupled quantum dots in the quantum Hall regime and showed (besides the splitting) that the peaks undergo shifts and also modulation as the magnetic field is continuously varied.

All the aforementioned experiments were usually performed by applying the same capacitive coupling (i.e., the same gate voltage) to each dot. The possibility of varying individually the voltages on each dot was realized by Hoffman et al. ${ }^{5}$ for parallel dots and by Kouwenhouven et al. ${ }^{1}$ for lateral dots, who obtained gray scale plots of the double-dot conductance as a function of the gate voltages $V_{g_{1}}, V_{g_{2}}$ applied on the dots. These plots are the so-called charging stability diagrams for double dots.

The quantum dot arrays are promising systems for mesoscopic physics because of the analogy with complex molecules and due to a possible realization of quantum bits (qubits) $^{6}$ (see also the review in Ref. 7 and the references therein). Another interesting topic is the observation of the Kondo effect in coupled quantum dots in both equilibrium and nonequilibrium regimes, as predicted theoretically., 8

Various theoretical attempts were made to describe the transport phenomena in coupled quantum dots at vanishing magnetic fields. The Hubbard-type approach initiated by Stafford and Das Sarma ${ }^{10}$ provided the evolution of the ad- dition spectrum of four CQD as the interdot tunneling is increased. Soon afterward, a master-equation technique was utilized $^{11}$ to predict that the conductance spectrum of a pair of CQD exhibits a twin-peak structure due to interdot coupling. A realistic electron-electron interaction was used but the approach failed in finding the saturation effect for strong couplings. There is also a series of papers ${ }^{12-14}$ which address the problem of CQD in the framework of constant capacitance model. The position and the shape of the conductance peaks in the Coulomb blockade regime were analyzed only in the limit of weak or strong tunneling between the dots. We mention that the tight-binding model has already been used for the study of transmittance of quantum dot arrays in the work of Kirczenow ${ }^{15}$ where each dot is associated with a single atom in the lattice, omitting thus the internal structure of the dots. Arrays of one-dimensional QDs were also studied by Stafford et al. ${ }^{16}$ and by Shangguan et al. ${ }^{17}$ Recently Das and $\mathrm{Rao}^{18}$ obtained theoretically the charging stability diagrams for a double dot modeled as one-dimensional Luttinger liquid with large barriers.

In this paper we address the problem of tunneling through arrays of CQD in a strong magnetic field. The specific phenomena we analyze are the multiplet formation, the effects of the intradot and interdot Coulomb interaction and strong magnetic field effects. Our theoretical approach is based on a non-Hermitian tight-binding (TB) Hamiltonian ${ }^{19}$ and the many-probe Landauer-Büttiker (LB) formalism. ${ }^{20,21}$ The non-Hermiticity arises when we include the contribution of the leads in the Hamiltonian of the dot system. The dot array is considered as a quantum-mechanically coherent system and we treat the electron-electron interactions beyond the usual constant capacitance model by describing both the intradot and interdot electron-electron interactions in a selfconsistent way. We recall that the constant capacitance model is actually not suitable for the study of quantum dots in the quantum Hall regime. ${ }^{22,23}$ Our formalism covers instead both the Coulomb blockade and the quantum Hall regimes. The calculations are performed at zero temperature. Since we are interested in the tunneling properties of artificial molecules in a strong magnetic field the dots are weakly coupled to the leads and the spins are frozen so that we neglect the Kondotype effects. 
In the present work each quantum dot in the array is described as a finite two-dimensional (2D) plaquette. Thus we pay attention to the internal structure of the dots in order to identify the effects of the magnetic field on the orbital motion of electrons within the dot and consequently on the transport properties of the array.

The rest of this paper is organized as follows. In Sec. II we outline the formalism through which we evaluate the transmittance of the coupled dot array system. In Sec. III we present our results and discuss their relevance to the experimental findings. While the numerical results are given for a particular array composed of two identical dots only we stress from the beginning that the description we give for the peak splitting and their saturation holds without any change for bigger arrays. We choose to study in more detail the double-dot case because the plots in this case are easier to follow. We conclude in Sec. IV.

\section{FORMALISM}

We consider an array of $N$ coupled (identical) quantum dots placed in a strong perpendicular magnetic field. The electronic transport through the structure is described by a generalization of the formalism used in Ref. 19 to describe the transport properties of a single QD. The Hamiltonian of a system of coupled dots contains the following terms:

$$
H^{N}=\sum_{k=1}^{N} H^{k}+\tau_{\text {int }} \sum_{k=1}^{N-1}\left(H_{t}^{k k+1}+\text { h.c. }\right)+U_{e} \sum_{k>k^{\prime}}^{N} H_{e e}^{k k^{\prime}},
$$

where $H^{k}$ is the Hamiltonian of $k$ th dot of the array which includes the intradot electron-electron interaction, $H_{t}^{k k+1}$ describes the tunnel coupling of consecutive dots, while $H_{e e}^{k k^{\prime}}$ is the operator that accounts for the Coulomb interaction between electrons located in different dots ( the so-called electrostatic interdot coupling). The 2D spinless Hamiltonian $H^{k}$ is considered in the self-consistent Hartree approximation (SCHA) and reads $\left(\left\langle i, i^{\prime}\right\rangle\right.$ denotes the nearest neighbor summation)

$H^{k}=\sum_{i \in Q D_{k}}\left(V_{g}^{k}+U \sum_{j(\neq i)} \frac{\left\langle n_{j}\right\rangle}{|j-i|}\right) c_{i}^{\dagger} c_{i}+t^{D} \sum_{\left\langle i, i^{\prime}\right\rangle} e^{i 2 \pi \phi_{i i^{\prime}}} c_{i}^{\dagger} c_{i^{\prime}}$.

Here $c_{i}^{\dagger}\left(c_{i}\right)$ are the creation (annihilation) operators in localized states indexed by $i \in Q D_{k}$ and $t^{D}$ is the nearestneighbor hopping integral in the dots. The phase $\phi_{i i^{\prime}}$ comes from the Peierls substitution and accounts for the magnetic flux through the unit cell of the lattice measured in quantum flux units $\phi / \phi_{0} .\left\langle n_{j}\right\rangle$ denotes the mean occupation number of the site $j$ from the $k$ th dot. Since we use a one particle approximation for the Coulomb repulsion which is restricted within the dots the Landauer-Büttiker formalism holds.

The charge transfer through the system is assured by several biased (noninteracting) leads attached at will to the quantum dot array. Following the idea formulated in Ref. 19 [namely Eq. (2.5)] we construct a non-Hermitian Hamiltonian $H_{\text {eff }}^{N}$ which acts only in the Hilbert space of the CQD system but takes into account the coupling of the array to the leads carrying the current. More explicitly, the lead-dot coupling will contribute to $H^{N}$ by the so-called "lead's selfenergy" (see Ref. 20 for a general discussion):

$$
H_{\mathrm{eff}}^{N}(E)=H^{N}+\tau^{2} \sum_{\alpha} e^{-i k} c_{\alpha}^{\dagger} c_{\alpha}, \quad E=2 \cos k,
$$

where $\tau$ is the strength of the lead-dot coupling and the index $\alpha$ denotes the sites where the leads are attached to the dots. We note that $H_{\text {eff }}^{N}$ depends on the energy $E$ of the incident electron and the imaginary part of its eigenvalues is controlled by the lead-dot coupling $\tau$. Finally, one replaces the effective Green function $G_{\text {eff }}^{+}(E)=\left(E-H_{\text {eff }}^{N}+i 0\right)^{-1}$ in the Landauer- Büttiker formula

$$
g_{\alpha \beta}\left(E_{F}\right)=\frac{e^{2}}{h} T_{\alpha \beta}\left(E_{F}\right)=4 \tau^{4} \sin ^{2} k\left|G_{\text {eff }, \alpha \beta}^{+}\left(E_{F}\right)\right|^{2}
$$

in order to obtain the conductance $g_{\alpha \beta}$, the transmittance $T_{\alpha \beta}$ and finally the Hall resistance as outlined previously. ${ }^{19} \mathrm{Re}-$ mark that $g_{\alpha \beta}$ depend as well on the gate voltages $V_{g}^{k}$ applied on each dot. Since the number of degrees of freedom of the terminals is infinite while the mesoscopic system has only a finite number of degrees of freedom, the Fermi level $E_{F}$ of the whole system is fixed by the leads.

The parameters entering our description are thus (i) the tunnel coupling between the dot system and the leads described by the parameter $\tau$, (ii) the interdot tunnel coupling $\tau_{\text {int }}$, (iii) the strength of the intradot and interdot Coulomb interaction $U$ and $U_{e}$, respectively, and (iv) the gate voltage $V_{g}^{k}$ which can be applied on any dot $k$. We take the hopping integral $t^{D}$ to be the unit of energy, so that all the other energies $V_{g}^{k}, \tau$, and $\tau_{\text {int }}$ are measured in units of $t^{D}$.

The calculation of $G_{\text {eff }}^{+}$must be performed numerically, and the mean occupation number of each site $\left\langle n_{i}\right\rangle$ in Eq. (2) must be calculated self-consistently. From Eq. (3), it is obvious that

$$
G_{\mathrm{eff}}(z)=\left[\left(G^{N}(z)\right)^{-1}-\tau^{2} \sum_{\alpha} e^{-i k} c_{\alpha}^{\dagger} c_{\alpha}\right]^{-1},
$$

so, for calculating $G_{\text {eff }}$ one needs to know the Green function $G^{N}=\left(z-H^{N}\right)^{-1}$ of the isolated CQD system. For large arrays this could be a difficult task (even for noninteracting dots) since the numerical effort depends on the number of dots and on the size of each dot. When the electrostatic interdot coupling is neglected (i.e., considering $U_{e}=0$ ), we found a recursive scheme which works efficiently for $N$ identical dots. This scheme expresses the Green function of the $N$-dot system $G^{N}$ in terms of $G^{N-1}$ and $G^{1}$, the Green function of a single isolated dot. To see this we use the perturbative ex-

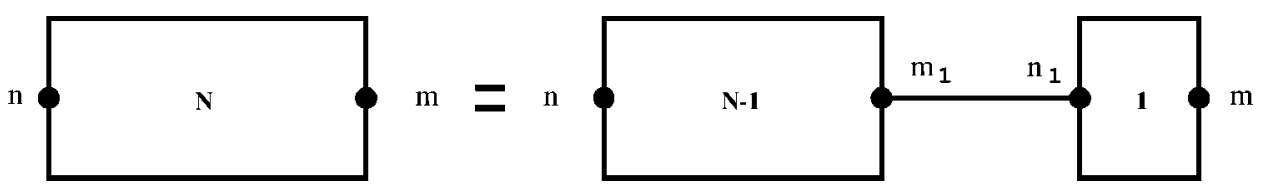

FIG. 1. The coupled dot system. 
pansion with respect to the coupling Hamiltonian which connects the group of $N-1$ dots with the single $N$ th dot (see Fig. $1)$. Briefly, one writes $H^{N}=H^{N-1}+H^{1}+\tau_{\text {int }}\left(H_{t}^{1, N-1}+H_{t}^{N-1,1}\right)$ $=H_{0}+\tau_{\text {int }} V$ and observes that $H_{0}$ and $G_{0}=\left(z-H_{0}\right)^{-1}$ are block diagonal matrices with respect to the following decomposi- tion of the $N$-dot Hilbert space $\mathcal{H}^{N}=\mathcal{H}^{N-1} \oplus \mathcal{H}^{1} . V$ is an offdiagonal perturbation (here $\mathcal{H}^{N-1}$ is the Hilbert space for $N$ -1 coupled dots and $\mathcal{H}^{1}$ is that for an isolated dot). The next step is to use a matrix form of the Dyson equation for $G^{N}(z)$ with respect to $V$ which reads

$$
G^{N}(z)=\left(z-H^{N}\right)^{-1}=G_{0}(z)\left(1-\tau_{\mathrm{int}} V G_{0}(z)\right)^{-1}=\left(\begin{array}{cc}
G^{N-1}(z) & 0 \\
0 & G^{1}(z)
\end{array}\right) \cdot \sum_{n \geqslant 0}^{\infty} \tau_{\mathrm{int}}^{n}\left(\begin{array}{cc}
0 & H_{t}^{N-1,1} G^{1}(z) \\
H_{t}^{1, N-1} G^{N-1}(z) & 0
\end{array}\right)^{n} .
$$

Then the matrix elements of $G^{N}$ can be calculated explicitly after summing the series in each subspace $\mathcal{H}^{N-1}$ and $\mathcal{H}^{1}$. Straightforward manipulations lead to the following system of equations:

$$
G_{\mathrm{nn}}^{N}(z)=G_{\mathrm{nn}}^{N-1}(z)+G_{\mathrm{nm}}^{N-1}(z) G_{\mathrm{nm}}^{N-1}(z) \frac{\tau_{\mathrm{int}}^{2} G_{\mathrm{nn}}^{1}(z)}{1-\tau_{\mathrm{int}}^{2} G_{\mathrm{mm}}^{N-1}(z) G_{\mathrm{nn}}^{1}(z)}
$$

and

$$
G_{\mathrm{nm}}^{N}(z)=G_{\mathrm{nm}}^{N-1}(z) G_{\mathrm{nm}}^{1}(z) \frac{\tau_{\mathrm{int}}}{1-\tau_{\mathrm{int}}^{2} G_{\mathrm{mm}}^{N-1}(z) G_{\mathrm{nn}}^{1}(z)} .
$$

Iterating Eq. (8) as many times as necessary we express the Green function of $N$-coupled quantum dots solely in terms of $G^{1}(z)$ [note that in the LB formula $G_{\mathrm{nm}}^{N}(z)$ is needed],

$$
G_{\mathrm{nm}}^{N}(z)=\tau_{\mathrm{int}}^{N-1}\left(G_{\mathrm{nm}}^{1}(z)\right)^{N} \prod_{i=1}^{N-1} \frac{1}{1-\tau_{\mathrm{int}}^{2} G_{\mathrm{nn}}^{1}(z) G_{\mathrm{mm}}^{1}(z) a_{i}(z)},
$$

where the complex coefficients $a_{i}$ are given by the recursion formula

$$
a_{1}=1, \quad a_{i}(z)=1+\frac{\tau_{\mathrm{int}}^{2}\left|G_{\mathrm{nm}}^{1}(z)\right|^{2} a_{i-1}}{1-\tau_{\mathrm{int}}^{2} G_{\mathrm{nn}}^{1}(z) G_{\mathrm{mm}}^{1}(z) a_{i-1}} \quad \text { for } \quad i \geqslant 2 .
$$

These formulas can be used to compute the electronic transmittance through any number of dots [a similar relation can be obtained for $G_{\mathrm{nn}}^{N}(z)$ which also makes the computation of occupation number $\left\langle n_{j}\right\rangle$ easier].

\section{RESULTS AND DISCUSSION}

Although the effects of the interdot coupling and electronelectron interaction competes in artificial molecules they must be considered separately. We start then with noninteracting dots and distinguish the charging effects later on.

As we have mentioned, we treat explicitly the simplest array, namely a double dot. Let us first discuss the spectral properties of double dots and their role in the resonant tun- neling. Figure 2(a) shows the spectrum of a double dot as a function of the magnetic flux. Each dot has $10 \times 10$ sites. As one can easily recognize the spectrum presents similarities with the Hofstadter spectrum of a mesoscopic plaquette (see, for instance, Fig. 2 in Ref. 24). As is well known, the eigenvalues from this spectrum can be labeled in terms of the topological properties of their eigenfunctions which are in turn of two types: edge and bulk states. The edge states are due to the Dirichlet boundary conditions and they fill the gaps existing in the spectrum of the same tight-binding Hamiltonian when periodic boundary conditions are used (see also Ref. 25). An important property of the edge states is that they are well separated in energy. In contrast, the energies of bulk states are grouped together.

The spectrum of the disconnected double dot is doubly degenerate and coincides with the usual spectrum of a 10 $\times 10$ plaquette. For coupled dots the new feature is that the spectrum exhibits a multiplet structure due to the tunnelcoupling $\tau_{\text {int }}$ between the dots which lifts the degeneracy of the energy levels. This degeneracy lifting and its dependence on $\tau_{\text {int }}$ will produce the peak splitting in the transmittance spectrum reported in the experiments. Indeed, through the Landauer-Büttiker formula [Eq. (4)], the transmittance spectrum as a function of the Fermi energy $E_{F}$ corresponds to the structure of the energy spectrum of the effective Hamiltonian [Eq. (3)]. The latter has resonances located near the eigenvalues of $H^{N}$ with the imaginary part controlled by the leaddot coupling $\tau$.

The correspondence between the transmittance peaks and the energy spectrum is shown in Figs. 2(a) and 2(b) and indicates the resonant tunneling process of the electron through the coherent double-dot structure represented schematically in Fig. 2(c). This correspondence depends essentially on the strength of the lead-array coupling, namely on $\tau$. For $\tau$ small the real part of the second term in $H_{\text {eff }}^{N}$ is small and gives only a small shift of the resonance. In contrast, a large $\tau$ spoils the resonant aspect and the one-to-one correspondence. Let us mention that for a flux $\phi / \phi_{0}=0.15$ and a plaquette of $10 \times 10$ sites, the ratio between the linear dimension of the system and cyclotron radius approximately equals 10.

We now discuss the subpeak aspect. Regarding Fig. 2(b) one notices that some doublets [the ones within the range $\left.V_{g} \in(2,3)\right]$ have a clear resolution and are rather equally 


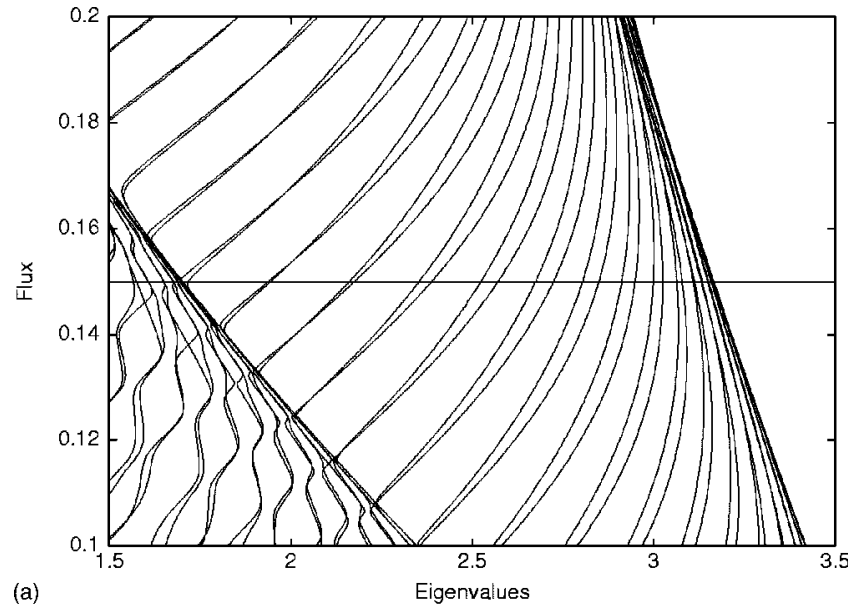

(a)

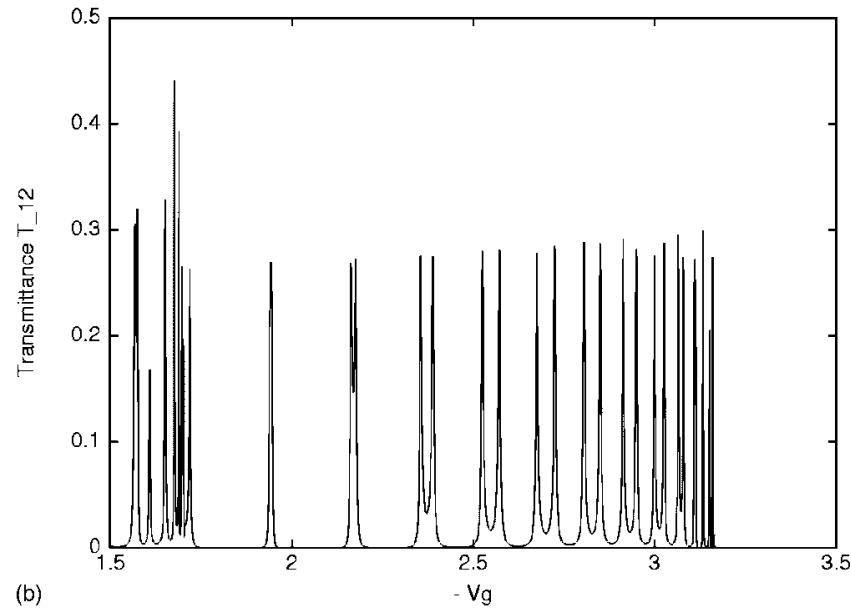

(b)

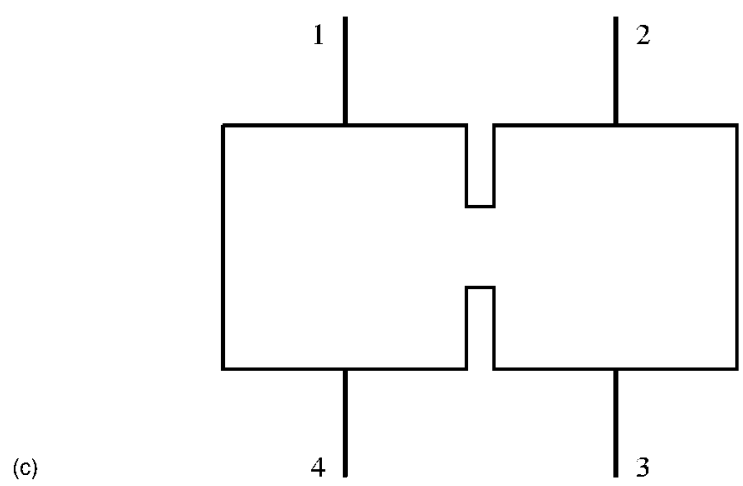

FIG. 2. (a) The degeneracy lifting of the eigenvalues for the Hofstadter-type spectrum of a noninteracting double dot; each dot consists of $10 \times 10$ sites and $\tau_{\text {int }}=0.4$. (b) The corresponding split peaks in the transmittance $T_{12}$ as a function of the gate potential at magnetic flux $\phi / \phi_{0}=0.15, \tau=0.4$, and $E_{F}=0$. (c) The sketch of the double dot with four terminals for which the transmittance has been calculated.

spaced. This happens because in a strong magnetic field the distance between two subpeaks is determined by the type of the corresponding resonant level (edge or bulk). To see this we first show in Fig. 3 the spatial distribution of the first and 29th eigenvectors of the double dot [the positions of the associated peaks in Fig. 2(b) are $V_{g}=3.1655$ and $V_{g}$ $=2.3880$, respectively]. Moreover, if the distance $\Delta$ between two initially degenerate bulk states is small the two subpeaks produced by the interdot coupling are mixed. Since the array is a coherent quantum system the eigenfunctions have the character of molecular states (i.e., the eigenfunctions are extended to both dots). When one of the dots is detuned (by applying an additional gate potential) the molecular states are scrambled with important effects on the orbital magnetization. ${ }^{26}$

A more interesting regime is the one in which the capacitive coupling is different $\left(V_{g 1} \neq V_{g 2}\right)$. As is well known experimentally, ${ }^{1}$ for two lateral QD's coupled in series the gray scale plot of $g_{12}\left(V_{g 1}, V_{g 2}\right)$ has a honeycomb pattern. In Fig. 4 we present stability diagrams for our double dot. The similarity with the calculations based on the constant capacitance model (CCM) in Ref. 1 and with the experimental gray scale plots in Ref. 5 is clearly seen. The similarity exists in spite of the fact that we are here in the strong magnetic field, while the above-mentioned stability diagrams are obtained in the absence of it. We observe that for almost decoupled dots [Fig. 4(a)] a chessboard pattern is obtained, which changes to the honeycomb picture at moderate coupling. A rather large $\tau_{\text {int }}$ deforms the hexagonal domains and at perfect coupling the diagram is reduced to a bunch of straight lines. We point out that in the strong magnetic field case a rather regular aspect of the rhomboids composing the stability diagram is found only in a range of gate voltages that contains peaks arising from edge states. Indeed, looking at Figs. 2(a) and 2(b) one may easily check that the interval $[2.55,2.95]$ satisfies this condition. Moreover, in the diagrams shown in Fig. 4 the traces lying on the line $V_{g 1}=V_{g 2}$ represent the usual subpeaks obtained at the same capacitive coupling on dots.

Our approach also reproduces the experimental results of Livermore et al.; ${ }^{4}$ Figs. 3(a)-3(c) in Ref. 4 reveal that, in a strong magnetic field, the transmittance of a double dot behaves as follows: (i) at a given magnetic field but increasing interdot coupling, one starts with single peaks [Fig. 3(a)], then twin peaks appear [Fig. 3(b)] and finally, at large interdot coupling, the saturation leads to individual peaks [Fig. 3(c)]; (ii) with increasing magnetic field the position of each peak shifts linearly (in spite of a zig-zag appearance) versus larger gate voltages. Both these features can be described by our model even without considering the interaction effects. Our results shown in Fig. 5 indicate a striking similarity to the experimental situation. We recover both the increase of the distance between the twin peaks by increasing the interdot tunneling $\tau_{\text {int }}$ and the drift of the peaks with the variation of the magnetic field. Again we found that such a regular behavior, as observed in the experiment, occurs whenever the Fermi level lies in a region covered by the edge states.

We now study the peak splitting in the case of interacting double dots. Figures 6(a)-6(d) show the Coulomb peaks of an artificial molecule composed of two $8 \times 8$ dots for different interdot couplings $\tau_{\text {int }}$. As expected, the splitting increases with $\tau_{\text {int }}$ and saturates at perfect coupling. The saturation is nonlinear in the coupling parameter $\tau_{\text {int }}$ which can be shown only by taking into account all orders of the perturbation as we make here. Again we remark the differences between edge and bulk subpeaks and for clarity we show in Fig. 7 the splitting process for a single edge subpeak and a bunch of bulk subpeaks [noticing that the $V_{g}$ interval in Figs. 7(a) and 7(b) have the same length, one can check that the 

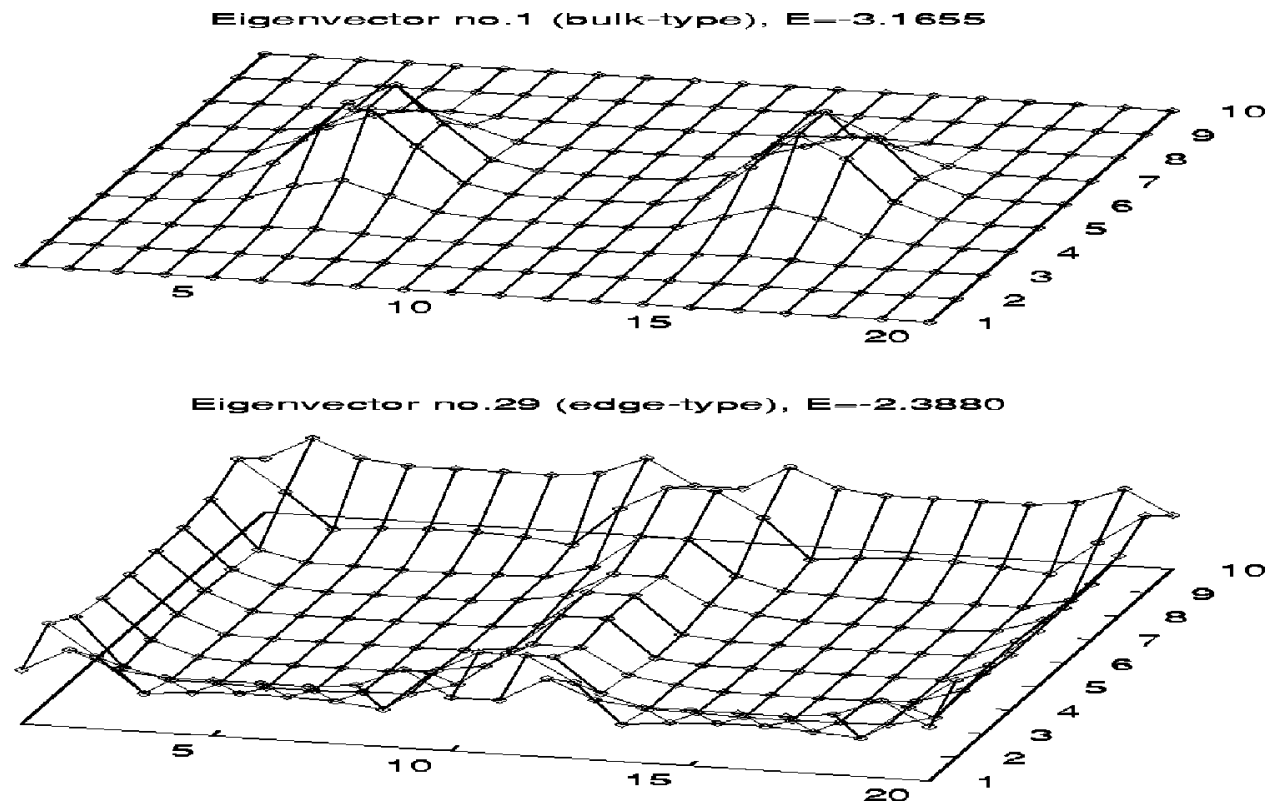

FIG. 3. The spatial distribution of the first and 29th eigenvectors inside a double dot composed of two identical dots $10 \times 10$ sites each. $\left(\phi / \phi_{0}=0.15, \tau_{\text {int }}=0.4\right)$. The first eigenvector is localized in the middle of each dot while the 29th is clearly stuck to the edges; note also the formation of a coherent molecular state due to the interdot coupling.

bulk states are denser and their splitting is more difficult to follow]. In what concerns the interaction effects it was shown for the single-dot problem that, at least in SCHA, the Coulomb repulsion gives rise to an increase in the level spacing and in the width of transmission peaks. ${ }^{19}$ The same features should hold also for coupled dots. To go beyond these qualitative statements we present in Fig. 8 the positions $V_{g}^{(i)}$ of two edge doublets $(i=9,10,11,12)$ as a function of the interaction strength $U$, at fixed interdot tunnel coupling. We identify two effects: (i) each subpeak position depends linearly on $U$ but with different slopes; the slopes increase (in absolute value) with the index $i$ as expected since at each subpeak the number of electrons increases, hence the Coulomb repulsion is enhanced. (ii) The splitting increases slowly with the interaction strength.

The results shown in Figs. 6 and 7 are obtained by taking into account the total Coulomb interaction. However, one asks usually how important the interdot interaction is, in par-
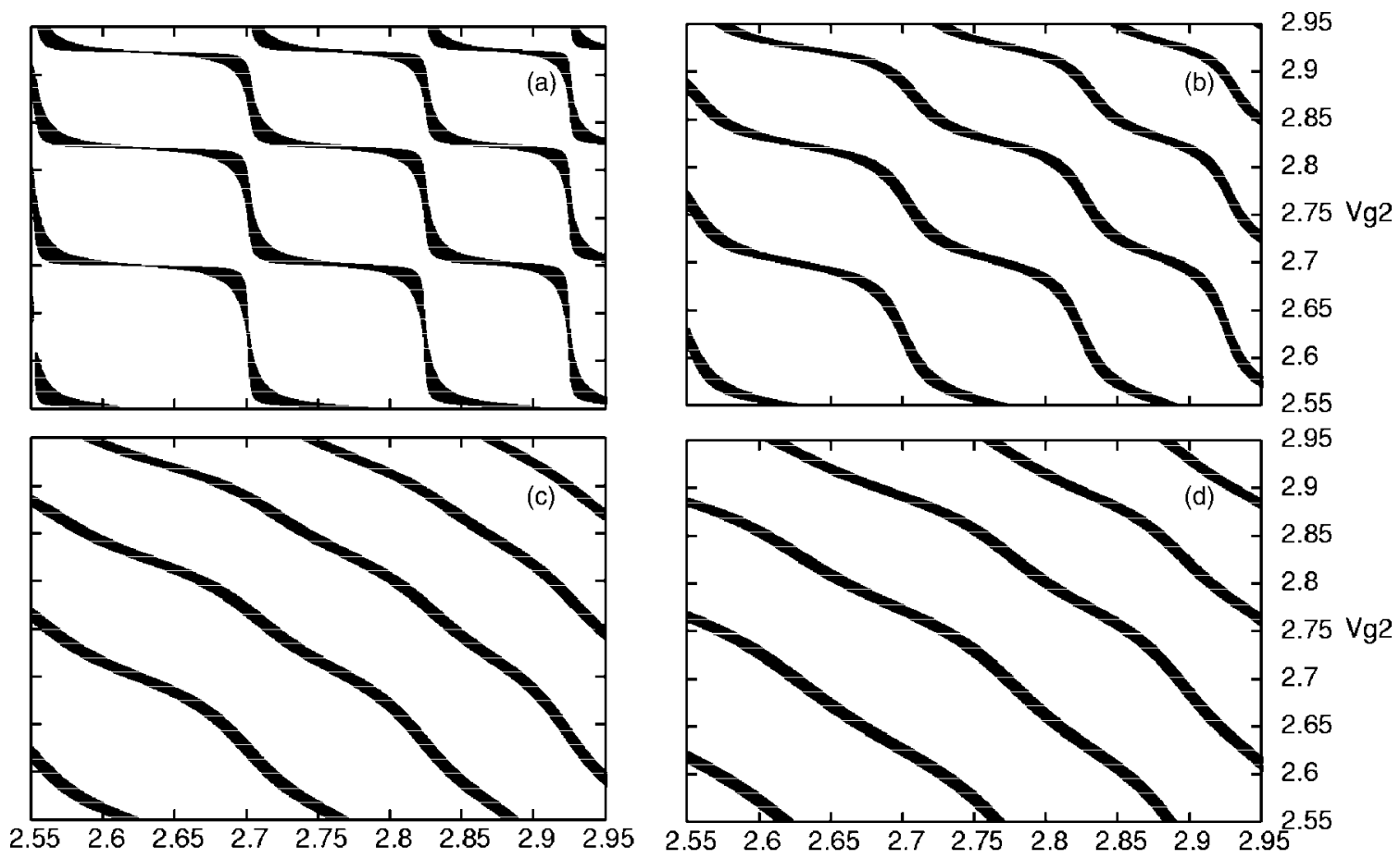

Vg1

Vg1

FIG. 4. Charging diagrams for a double dot in the strong magnetic field $\left(\phi / \phi_{0}=0.15, \tau=0.4\right)$. (a) $\tau_{\text {int }}=0.2$, (b) $\tau_{\text {int }}=0.4$, (c) $\tau_{\text {int }}=0.6$, (d) $\tau_{\text {int }}=1$. 

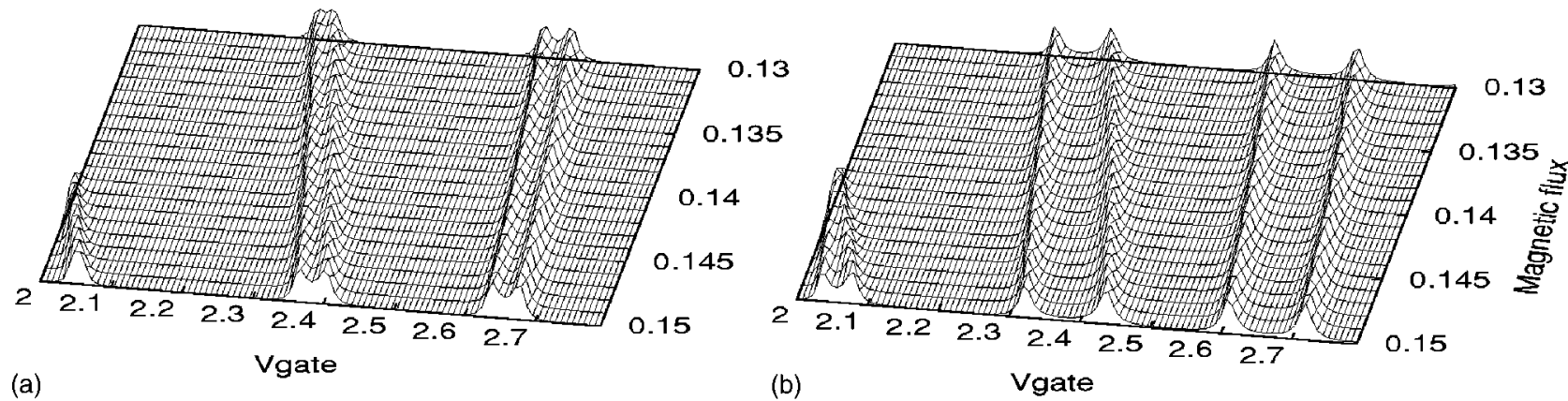

FIG. 5. The drift with the magnetic field of the twin peaks from the conductance spectrum of a noninteracting double dot $(5 \times 8$ sites per dot) for two values of the tunnel coupling: (a) $\tau_{\text {int }}=0.2$, (b) $\tau_{\text {int }}=0.6$. For both cases $E_{F}=0$.

ticular when the dots are small. This contribution represents an additional term compared to the intradot Coulomb repulsion, and it was suggested that the strength $U_{e}$ of the interdot interaction is smaller than the intradot interaction strength $U$ because of the screening produced by the metallic gates between neighboring dots. ${ }^{27}$

The interdot term should accentuate the interaction effects observed in the transmission through a single dot. Furthermore, since the interaction energy is positive, in the presence of the additional interdot electrostatic coupling the whole transmittance spectrum is pushed upward on the energy scale, corresponding to smaller gate voltages. This is noticed by comparing Figs. 9(a) and 9(b) which shows the transmit- tance spectrum of a double dot composed of two dots having $5 \times 8$ sites each with and without the interdot interaction. The latter increases the width of the peaks even further, leading also to a reduction in the depth of the multiplets. This explains the poor resolution of the doublet around $V_{g}=2.35$ shown in Fig. 9(a).

The progressive character of the level splitting and the contribution of the interaction in comparison to the noninteracting case is shown in Fig. 10 for three well-defined doublets in the region of edge states. Figure 10(a) presents our results for the transmittance and the number of electrons accommodated in the double-dot system as a function of the gate voltage and reveals the addition of two electrons at each
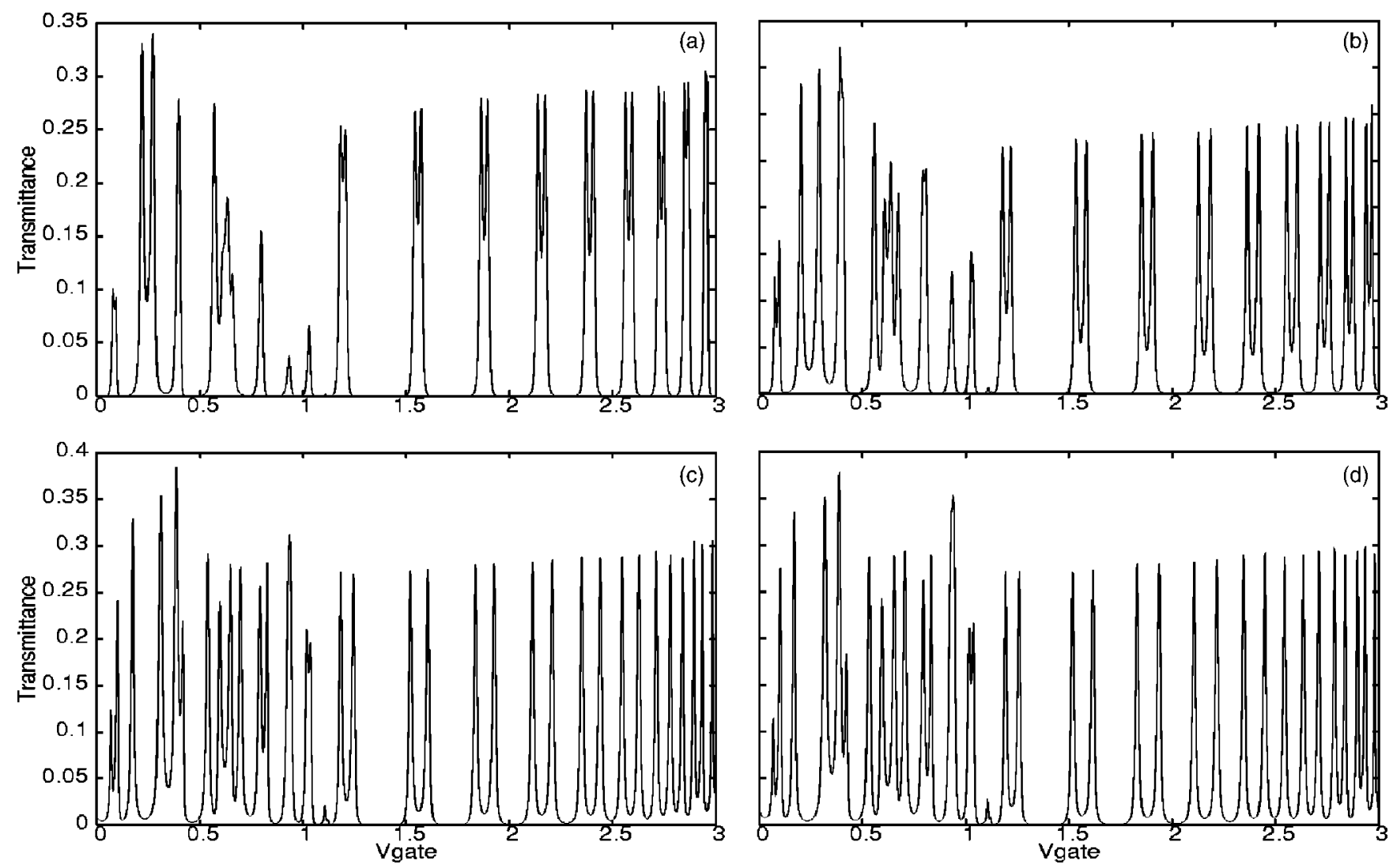

FIG. 6. The dependence of the transmittance spectrum of a double dot (having $8 \times 8$ sites per dot) on the interdot coupling $\tau_{\text {int }}$ at fixed interaction strength $U=U_{e}=0.1$ and $\phi / \phi_{0}=0.15, E_{F}=0$. (a) $\tau_{\text {int }}=0.2$, (b) $\tau_{\text {int }}=0.4$, (c) $\tau_{\text {int }}=0.8$, (d) $\tau_{\text {int }}=1$. The peaks behavior reproduces the experimental observations; a usual peak is tunnel split into a multiplet and the splitting increases with $\tau_{\text {int }}$. 

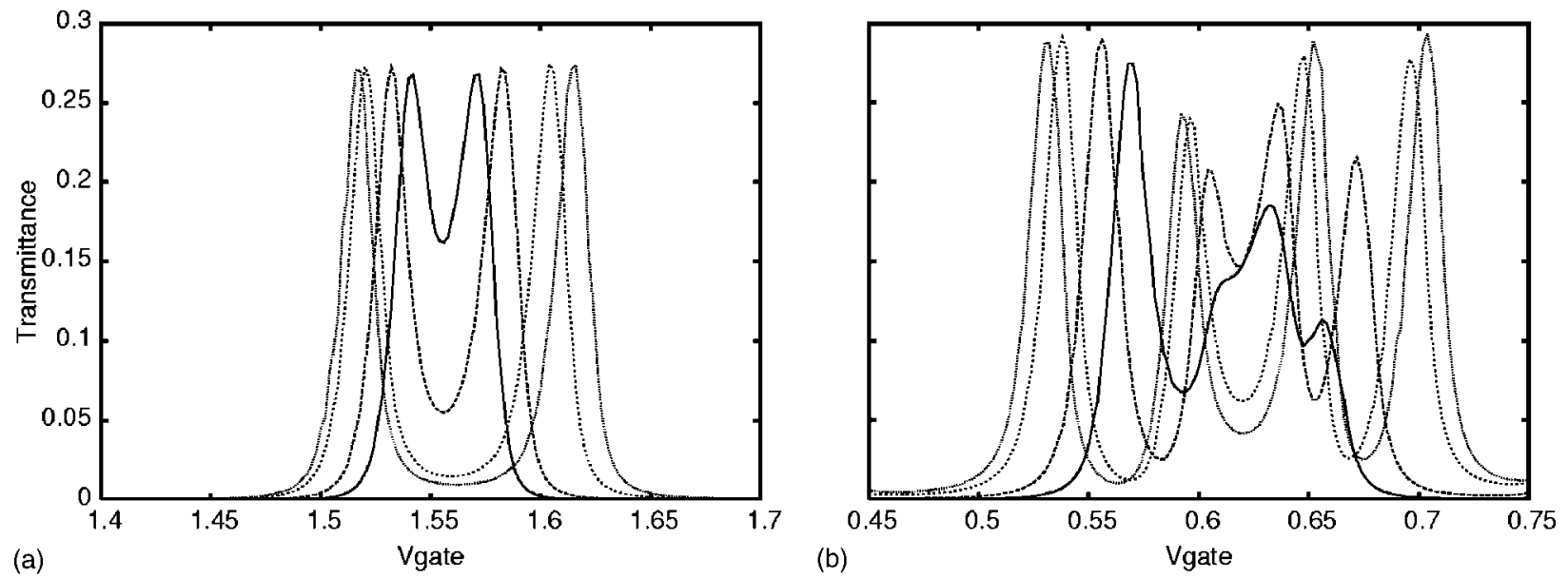

FIG. 7. The peak splitting at different values of $\tau_{\text {int }}$ for edge (a) and bulk (b) subpeaks: $\tau_{\text {int }}=0.2$ ( solid line), $\tau_{\text {int }}=0.4$ (long-dashed line), $\tau_{\text {int }}=0.8$ (dashed line), and $\tau_{\text {int }}=1$ (dotted line).

doublet. This represents the so-called bunching effect which means that two (or more) electrons are added almost simultaneously within the dots; in our case the bunching is due to the interdot coupling and is present as long as the saturation has not yet set in. Figure 10(b) shows the magnitude of the splitting $\Delta V_{n}(n=1,2,3)$ as a function of the interdot coupling $\tau_{\text {int }}$ at $U=U_{e}=0.5$ and $U=U_{e}=0$ and evidently demonstrates that (i) $\Delta V_{n}$ depends on the peak index $n$, i.e., different peaks have different robustness to splitting; (ii) $\Delta V_{n}$ is progressive with $\tau_{\text {int }}$; the slope of $\Delta V_{n}$, while staying positive, decreases with $\tau_{\text {int }}$; (iii) the presence of the Coulomb interaction gives rise to a larger splitting in $\Delta V_{n}$ and to a more rapid saturation compared to noninteracting case. (We draw the splitting as function of $\tau_{\text {int }}^{2}$ in order to keep the analogy with experimental curves which are presented in terms of internal conductance $G_{\text {int }}^{2}$.)

The peak structure of the transmittance (conductance) matrix impose specific behavior on the Hall resistance. More precisely any minimum of the conductance gives rise to a maximum in $R_{H}$, according to the formula

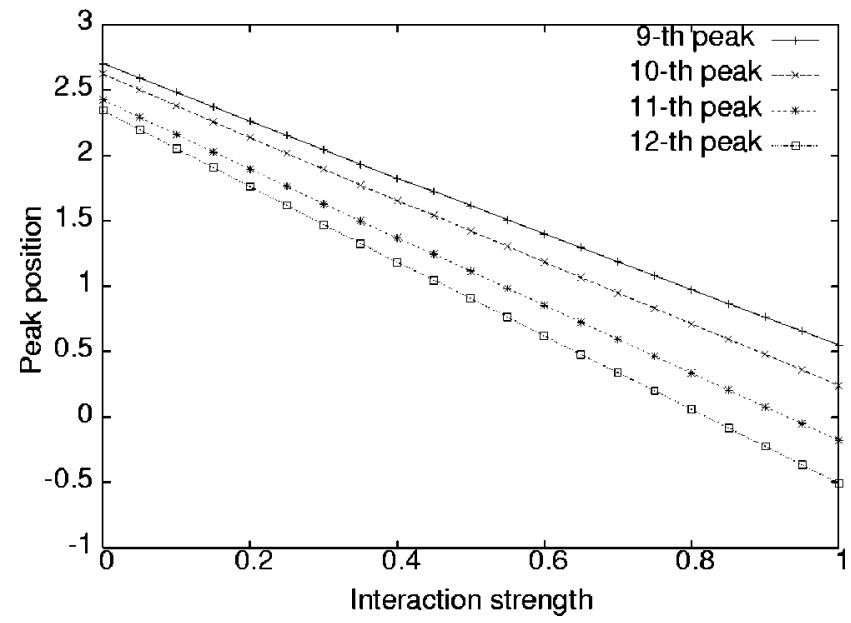

FIG. 8. The peak positions $V_{g}^{(i)}$ of two edge doublets from the transmittance spectrum of a double dot $(8 \times 8$ sites per dot $)$ as a function of the interaction strength $U\left(U=U_{e}, i=9,10,11,12, \tau_{\text {int }}\right.$ $\left.=0.4, E_{F}=0\right)$. The distance between the doublets as well as their splittings increase smoothly with $U$.

$$
R_{H}=\left(g_{21} g_{43}-g_{12} g_{34}\right) / D,
$$

$D$ being a $3 \times 3$ subdeterminant of the matrix $g_{\alpha \beta}$. Obviously, the minima of the conductance occur in-between the resonances and in the same place the maxima of the Hall resis-

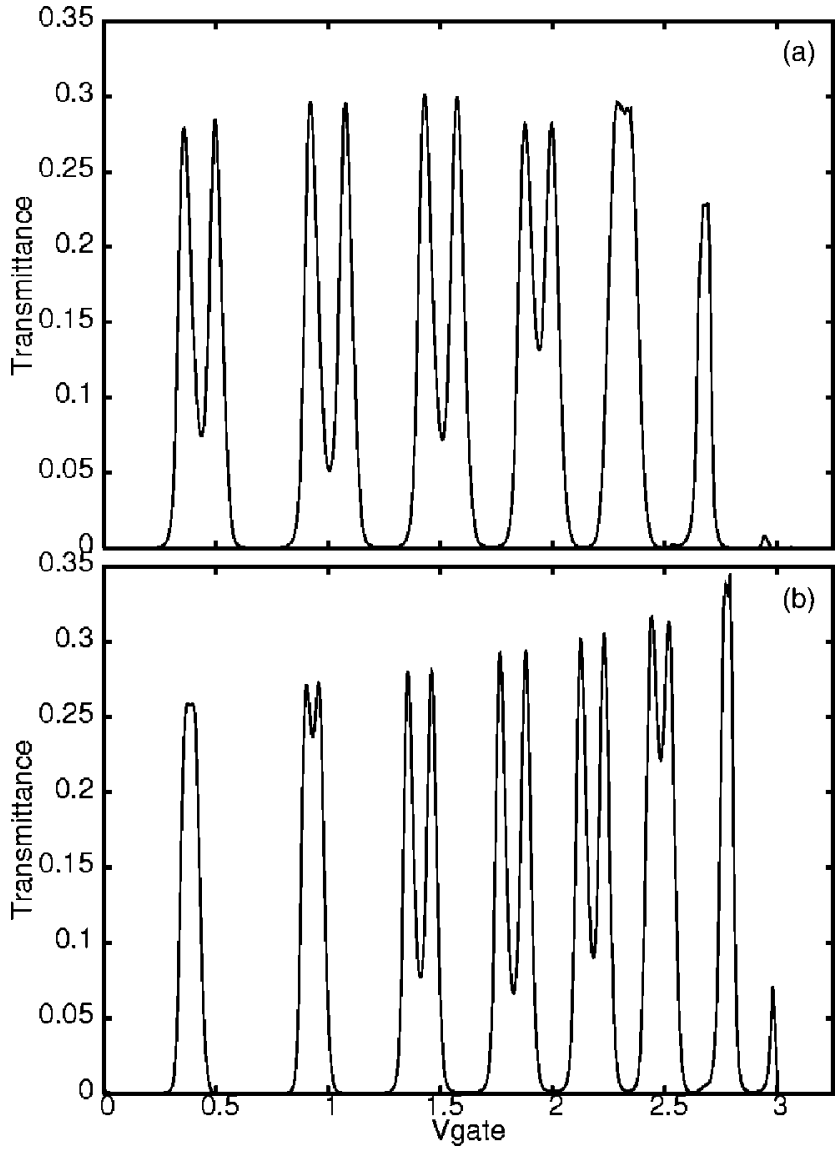

FIG. 9. Contribution of the interdot Coulomb interaction: (a) Transmittance spectrum in the presence of both interdot and intradot interaction $\left(U=U_{e}=0.5\right)$. (b) The same in the presence of intradot electron-electron interaction only $\left(U=0.5, U_{e}=0\right)$. The parameters are: $\tau_{\text {int }}=0.2, \tau=0.4, E_{F}=0$. 

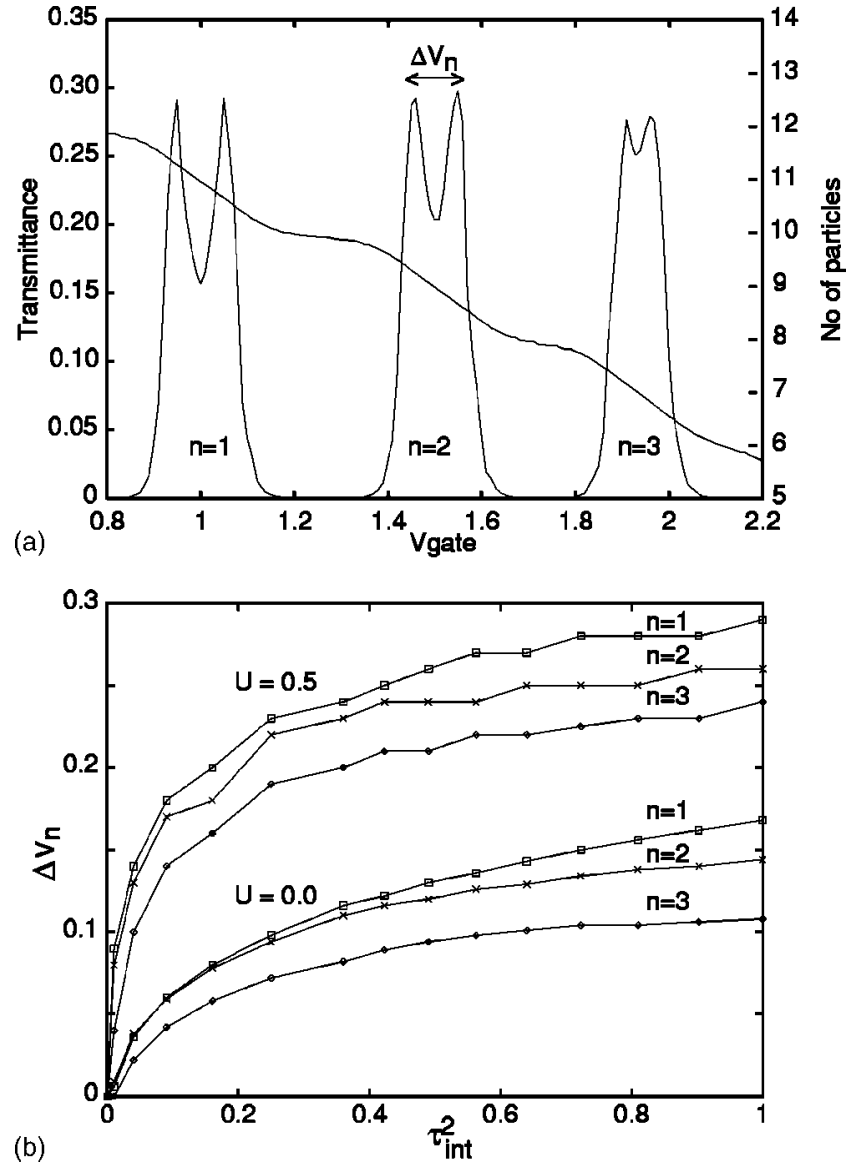

FIG. 10. (a) Three split peaks corresponding to the edge states, mapped onto the electronic occupation number calculated for $\tau$ $=0.4$ and $\tau_{\text {int }}=0.1\left(U=U_{e}=0.5, E_{F}=0\right)$. The rather smooth curve of the number of particles indicates that the charge quantization is somewhat poor, nevertheless it can be observed that at each doublet the number of electrons increases by two. (b) The magnitude of splitting $\Delta V_{n}$ versus the interdot coupling for the three doublets from above, for both non-interacting $\left(U=U_{e}=0\right)$ and interacting case $\left(U=U_{e}=0.5\right)$.

tance can be found, resulting in an oscillating behavior of the Hall resistance. Such a behavior was put into evidence for single dots in in the case of pinched contacts to the leads. ${ }^{19}$ In the present case of multiple dots, the interdot coupling should add its own pattern because the splitting of the energy levels gives rise to a richer aspect of the transmittance spectrum. In Fig. 11 we show plots of the Hall resistance for an interacting double dot at different interdot couplings. One notices that at small $\tau_{\text {int }}$ [Fig. 11(a)] there are two types of oscillations that correspond to the doublet structure of the resonances. The large amplitude oscillations correspond to the minima between two doublets while the small oscillations are due to the local minima found between two peaks composing a doublet. By increasing the interdot coupling (and hence the splitting) the local minima get smaller; consequently the amplitude of the $R_{H}$ oscillations is enhanced and at perfect coupling [Fig. 11(c)] all maxima become comparable. In this case the oscillation pattern resembles the one of a single (bigger) dot [compare for instance Figs. 11(c) and
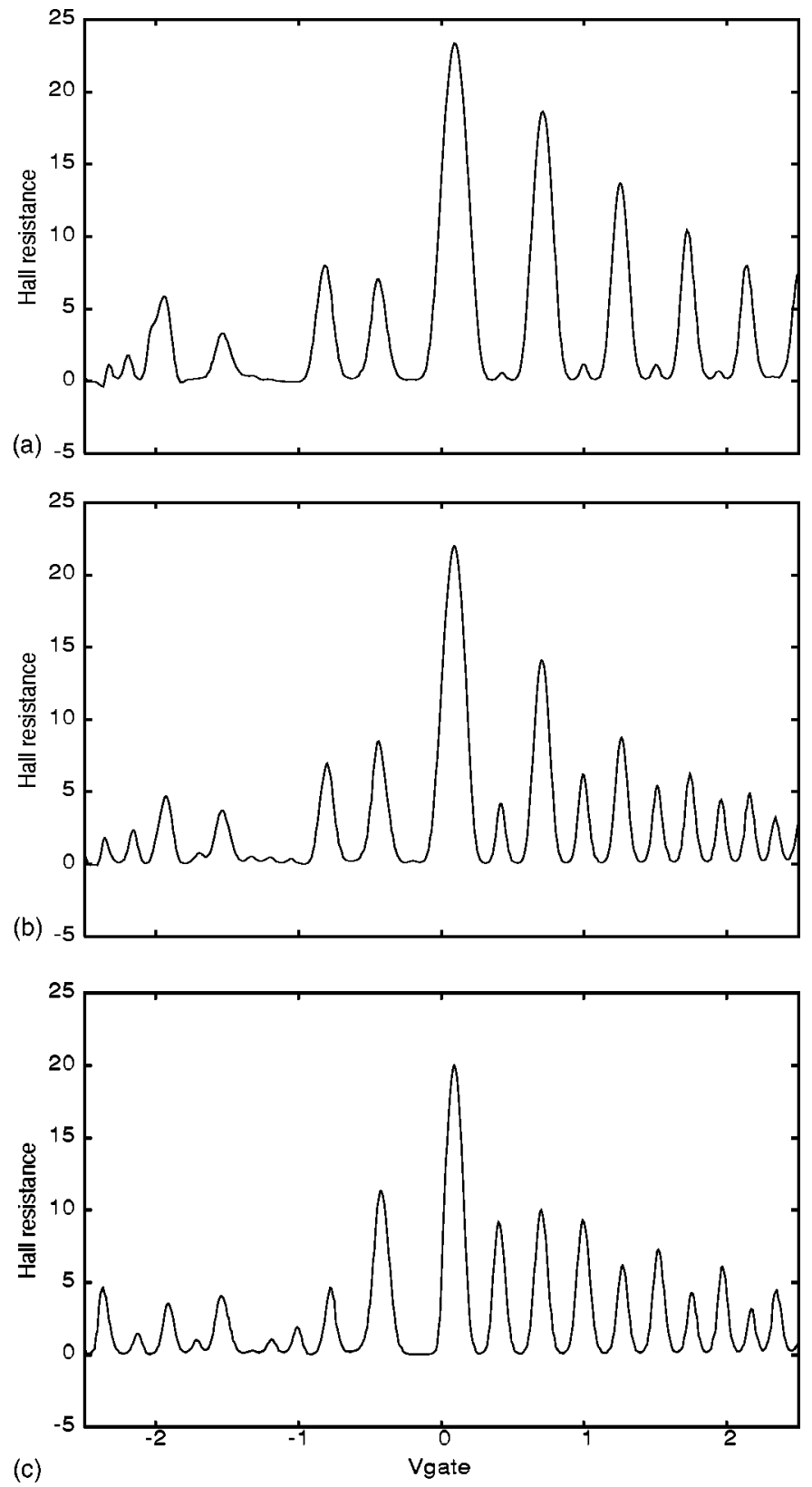

FIG. 11. The oscillatory behavior of the Hall resistance for an interacting double dot in high magnetic field regime $\left(U=U_{e}=0.5\right.$, $\left.\phi / \phi_{0}=0.15, \tau=0.4\right)$ for several interdot tunnel couplings. (a) $\tau_{\text {int }}$ $=0.2$, (b) $\tau_{\text {int }}=0.6$, (c) $\tau_{\text {int }}=1$. The small amplitude oscillations due to the splitting are more evident in the range $V_{g}>0$ where the spectrum is covered by edge states. The same gate potential is applied on both dots and $E_{F}=0$.

6(b) from Ref. 19]. To the best of our knowledge such a property has not yet been observed experimentally.

Finally, as an illustration of our recursive method we present in Fig. 12 the multiple subpeak structure of the transmittance through a chain of 10 dots, together with its phase evolution as a function of the plunger gate voltage, which shows sudden jumps by $\pi$ between each subpeak of the multiplet. 


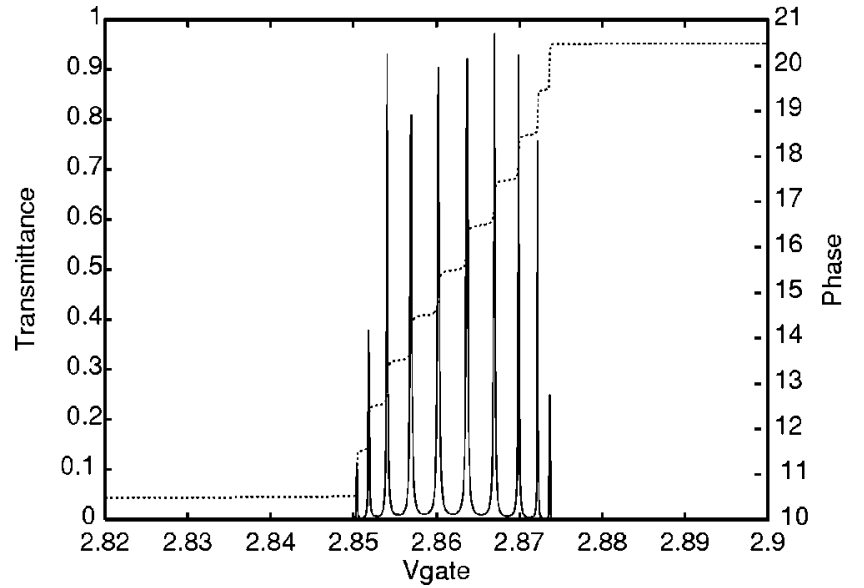

FIG. 12. Multiplet in the transmittance spectrum of an array of 10 dots $(5 \times 8$ sites each $)$ and the transmission phase as a function of the gate potential in the strong field regime $\phi / \phi_{0}=0.15$. The transmittance is measured in units of $e^{2} / h$ and the phase in units of $\pi$. The phase increases by $\pi$ on each subpeak of the multiplet. Other parameters are $\tau=0.2, \tau_{\text {int }}=0.2$.

\section{CONCLUSIONS}

The Landauer-Büttiker approach has been used to describe the electronic transport through an array of coupled quantum dots placed in a strong perpendicular magnetic field. The formalism permits to consider the parameters which determine the problem (interdot coupling $\tau_{\text {int }}$ and leaddot coupling $\tau$ ) in all orders of the perturbation and thus to obtain results in the whole range of variation of these parameters. We have shown that the multiplet structure is determined by the tunnel coupling between the dots, while the Coulomb interaction gives rise to a significant additional width of the peaks and increases the splitting. The latter saturates at perfect interdot coupling. The strong magnetic field leads to specific features summarized as follows. (i) The splitting depends on the nature of the states from which it evolves (bulk or edge). States show different robustness to splitting. (ii) At small and moderate interdot couplings, one obtains weak oscillations in the Hall resistance in addition to the usual ones. (iii) The position of the peaks (and subpeaks) shifts with increasing magnetic field; this yields a diagram (Fig. 5) which is similar to what was found experimentally by Livermore et al. ${ }^{4}$

Finally, the stability diagrams of double dots in strong magnetic field were reproduced. As shown by Holleitner et $a l .^{28}$ the investigation of such diagrams for a double-dot embedded in a mesoscopic ring is the first step in understanding the quantum transport through Aharonov-Bohm interferometers with quantum dots. A theoretical approach to this topic will be explored elsewhere.

\section{ACKNOWLEDGMENTS}

This work was supported by Grant No. CNCSIS/2002 and Romanian Programme for Fundamental Research. V.M. acknowledges support from the NATO-TUBITAK and the Romanian Ministry of Education and Research under CERES contract. B.T. acknowledges the support of TUBITAK, NATO-SfP, MSB-KOBRA, and TUBA.
${ }^{1}$ W. G. van der Wiel, S. de Francheschi, J. M. Elzerman, T. Fujisawa, S. Tarucha, and L. P. Kouwenhoven, Rev. Mod. Phys. 75, 1 (2003).

${ }^{2}$ F. R. Waugh, M. J. Berry, D. J. Mar, R. M. Westervelt, K. L. Campman, and A. C. Gossard, Phys. Rev. Lett. 75, 705 (1995).

${ }^{3}$ F. R. Waugh, M. J. Berry, C. H. Crouch, C. Livermore, D. J. Mar, R. M. Westervelt, K. L. Campman, and A. C. Gossard, Phys. Rev. B 53, 1413 (1996).

${ }^{4}$ C. Livermore, D. S. Duncan, R. M. Westervelt, K. D. Maranowski, and A. C. Gossard, Phys. Rev. B 59, 10744 (1999); J. Appl. Phys. 86, 4043 (1999).

${ }^{5}$ F. Hofmann, T. Heinzel, D. A. Wharam, J. P. Kotthaus, G. Böhm, W. Klein, G. Trankle, and G. Weimann, Phys. Rev. B 51, 13872 (1995).

${ }^{6}$ D. Loss and D. P. DiVincenzo, Phys. Rev. A 57, 120 (1998).

${ }^{7}$ D. P. DiVincenzo, G. Burkard, D. Loss, and E. V. Sukhorukov, in Quantum Mesoscopic Phenomena and Mesoscopic Devices in Microelectronics, edited by I. O. Kulik and R. Ellialtioglu (Kluwer, Dordrecht, 2000), p. 399.

${ }^{8}$ T. Aono and M. Eto, Phys. Rev. B 64, 073307 (2001).

${ }^{9}$ R. Aguado and D. C. Langreth, Phys. Rev. B 67, 245307 (2003).

${ }^{10}$ C. A. Stafford and S. Das Sarma, Phys. Rev. Lett. 72, 3590 (1994).

${ }^{11}$ G. Klimeck, G. Chen, and S. Datta, Phys. Rev. B 50, 2316
(1994).

${ }^{12}$ K. A. Matveev, L. I. Glazman, and H. U. Baranger, Phys. Rev. B 53, 1034 (1996); 54, 5637 (1996).

${ }^{13}$ J. M. Golden and B. I. Halperin, Phys. Rev. B 53, 3893 (1996); 54, 16757 (1996).

${ }^{14}$ R. Ziegler, C. Bruder, and H. Schoeller, Phys. Rev. B 62, 1961 (2000).

${ }^{15}$ G. Kirczenow, Phys. Rev. B 46, 1439 (1992).

${ }^{16}$ C. A. Stafford, R. Kotlyar, and S. Das Sarma, Phys. Rev. B 58, 7091 (1998).

${ }^{17}$ W. Z. Shangguan, T. C. Au Yeung, Y. B. Yu, and C. H. Kam, Phys. Rev. B 63, 235323 (2001).

${ }^{18}$ S. Das and S. Rao, Phys. Rev. B 68, 073301 (2003).

${ }^{19}$ V. Moldoveanu, A. Aldea, A. Manolescu, and M. Niță, Phys. Rev. B 63, 045301 (2001).

${ }^{20}$ S. Datta, Electronic Transport in Mesoscopic Systems (Cambridge University Press, Cambridge, 1995), p. 146.

${ }^{21}$ M. Büttiker, in Nanostructured Systems, edited by M. Reed [Semicond. Semimetals 35, 191 (1992)].

${ }^{22}$ L. P. Kouwenhouven et al., in Mesoscopic Electron Transport, NATO ASI Series E, Vol. 345, edited by L. L. Sohn, L. P. Kouwenhouven, and G. Schön (Kluwer, Dordrecht, 1997).

${ }^{23}$ P. L. McEuen, E. B. Foxman, J. Kinaret, U. Meirav, M. A. Kastner, N. S. Wingreen, and S. J. Wind, Phys. Rev. B 45, 11419 
(1992).

${ }^{24}$ U. Sivan, Y. Imry, and C. Hartzstein, Phys. Rev. B 39, 1242 (1989).

${ }^{25}$ A. Aldea, P. Gartner, A. Manolescu, and M. Niță, Phys. Rev. B 55, R13 389 (1997).

${ }^{26}$ A. Aldea, V. Moldoveanu, M. Niță, A. Manolescu, V. Gudmundsson, and B. Tanatar, Phys. Rev. B 67, 035324 (2003).
${ }^{27}$ F. Ramirez, E. Cota, and S. E. Ulloa, Phys. Rev. B 59, 5717 (1999).

${ }^{28}$ A. W. Holleitner, C. R. Decker, H. Qin, K. Eberl, and R. H. Blick, Phys. Rev. Lett. 87, 256802 (2001); A. W. Holleitner, H. Qin, K. Eberl, and J. P. Kotthaus, Physica E (Amsterdam) 12, 774 (2002). 